\title{
Localizing Putative Markers in Genetic Association Studies by Incorporating Linkage Disequilibrium into Bayesian Hierarchical Models
}

\author{
Brooke L. Fridley Gregory D. Jenkins \\ Division of Biomedical Statistics and Informatics, Department of Health Sciences Research, Mayo Clinic College of \\ Medicine, Rochester, Minn., USA
}

\section{Key Words}

Genetic association - Linkage disequilibrium • Multi-SNP

models · Shrinkage · Single nucleotide polymorphisms • SNPs

\begin{abstract}
Objective: Numerous methods have been proposed to model the association between multiple single nucleotide polymorphisms (SNPs) and a phenotype. Often these methods do not explicitly model the information regarding the linkage disequilibrium (LD) between SNPs. Furthermore, many methods shrink the SNP effects towards zero, rather than to an unknown latent gene-level effect. Methods: We outline the use of bayesian hierarchical models for gene-level analysis that incorporates LD information. Four different bayesian models, with either the inclusion or exclusion of LD information and 'shrinkage' of SNP effects to a latent genelevel effect or zero, were applied to a pharmacogenomic study and simulation study. Results: We observed that the inclusion of LD information resulted in increased precision in the SNP parameter estimates. The simulation study also demonstrated that the bayesian models were able to determine the simulated 'causative' variant more often with less false-positive associations as compared to commonly used multi-SNP analysis methods. Conclusions: Incorporating LD information in the analysis of multiple SNPs results in more
\end{abstract}

precise estimates of SNP effects. In addition, the bayesian models are able to isolate the simulated 'causative' variant more often than commonly used regression modeling methods.

Copyright $\odot 2010$ S. Karger AG, Basel

\section{Introduction}

Genetic association studies have recently become very popular for the study of complex diseases, which has led to an increased interest in 'individualized medicine' [1, 2]. In addition to the increase in the number of genetic association studies for complex diseases [3], interest in pharmacogenomics and the genetic basis for variation in drug response has also increased [4-9]. Many of these genetic association studies involve a set of candidate genes where a set of single nucleotide polymorphism (SNP) markers are genotyped within a gene. Numerous statistical methods have been proposed to model the effects of multiple SNPs simultaneously. Recently, several shrinkage and variable selection methods have been proposed for multi-SNP models which deal with the highdimensional aspect and multicollinearity or linkage disequilibrium (LD) between SNPs. Some of the commonly used shrinkage or variable selection methods are: ridge regression [10], lasso [11], bayesian variable selection [12- 
14], hierarchical, multilevel or mixed modeling [15-17], and hierarchical modeling involving a spatial covariance structure to model the linkage disequilibrium between SNP markers [18].

When shrinkage or variable selection methods are used with SNP data, often the information regarding the LD between SNPs is not explicitly reflected in the model. Recently, a hidden Markov random field model with a Dirichlet prior and a weighted LD graph was proposed to incorporate LD information between SNPs in the statistical analysis and was demonstrated to have increased sensitivity in identifying SNPs associated with the phenotype [19]. Therefore, the direct modeling of LD information between SNPs may increase power to detect markers relevant to the phenotype. In addition to excluding LD information in the statistical model, many 'shrinkage' analysis methods shrink the SNP effects towards zero, rather than to an unknown latent gene-level effect. By including a latent gene-level effect in the model, SNP effects are allowed to shrink to a common 'gene-level' effect that differs from zero. Furthermore, including a genelevel effect allows for testing of a gene effect.

Many of the current approaches rely on a nonbayesian viewpoint of 'shrinkage' and variable selection, such as ridge regression [10] or lasso [11]. A bayesian formulation of a regression model with a multivariate normal prior distribution of the SNP effects, $\beta \sim \operatorname{MVN}\left(\mathbf{0},\left(\sigma^{2} / k\right) \mathbf{I}\right)$, can be shown to be identical to ridge regression [20] or an L2 penalty. Likewise, a Laplace or double-exponential prior distribution on the SNP effects is equivalent to lasso regression [11] or an L1 penalty. Switching from a frequentist formulation to a bayesian one comes with many advantages: flexibility in model choice, ability to model dependency between parameters, possible inclusion of prior knowledge, and estimation of the joint distribution of all parameters simultaneously [21]. Lastly, within a bayesian setting we can easily allow for uncertainty in model form using bayesian model averaging approaches $[12,13,22-$ 25].

We present bayesian hierarchical models for the analysis of multiple SNPs, which incorporate LD information. Four different models are outlined and applied to a candidate gene pharmacogenomic study for the drug gemcitabine, as well as data from a simulation study involving different levels of LD, number of SNP markers, and 'causative' variants. The four models differ with respect to inclusion of LD information in the model, and shrinkage of the SNP effects towards zero or towards a non-zero latent gene-level effect.

\section{Methods and Materials}

Hierarchical Model

Let $Y_{i}$ represent the continuous phenotype for subject $i, i=$ $1, \ldots, N$, and let $X_{i j}$ represent the genotype for SNP $j, j=1, \ldots, K$, for subject $i$ representing the number of minor alleles observed (i.e. $X_{i j}=0,1$ or 2$)$. Next, $Y_{i}$ is modeled as $Y_{i} \sim \operatorname{Normal}\left(\mu_{i}, \sigma^{2}\right)$ with

$$
\mu_{i}=\delta+\sum_{j=1}^{k} \beta_{j} X_{i j}
$$

with $\beta_{j}, j=1, \ldots, K$ representing the SNP effects. Note that in the formulation of $\mu_{i}$ the effects of individual SNPs are assumed not to be a mixture of negative and positive effects. To achieve this, a certain level of preprocessing of the data to recode alleles will be required. The first model shrinks the SNP effects towards zero with the multivariate normal prior $\boldsymbol{\beta} \sim \operatorname{MVN}\left(\mathbf{0}, \tau^{2} \mathbf{I}\right.$ ) (model 1). The second model has the SNP effects shrunk towards a nonzero latent gene effect with the multivariate normal prior $\boldsymbol{\beta} \sim$ $\operatorname{MVN}\left(\alpha \times \mathbf{1}, \tau^{2} \mathbf{I}\right)$, where $\alpha$ represents the latent gene effect (model 2). For both models, diffuse proper prior distributions were specified for the remaining parameters in the model [i.e. $\sigma^{2} \sim$ Inverse $\operatorname{Gamma}(0.001,0.001), \tau^{2} \sim$ Inverse $\operatorname{Gamma}(0.001,0.001)$, $\delta \sim \operatorname{Normal}(0,1,000)]$. Inferences for individual SNP effects and the gene-level effect are based on the corresponding posterior distributions. For a discrete phenotype, a latent variable model [26] or generalized linear logistic/probit model could be utilized.

\section{Hierarchical Model Including LD Information}

The third and fourth models are similar to the models in the previous section, with the addition of LD information explicitly modeled. The third model shrinks the SNP effects towards zero with the LD modeled in the covariance matrix using the multivariate normal prior $\beta \sim \operatorname{MVN}\left(\mathbf{0}, \tau^{2} \mathbf{\Sigma}\right)$, where $\Sigma_{i j}$ is the correlation between SNP $i$ and SNP $j$ (model 3). The last model includes both the LD information and a latent gene effect with the prior for the SNP effects modeled as $\beta \sim \operatorname{MVN}\left(\alpha \times 1, \tau^{2} \Sigma\right.$ ) (model 4). Similar to models 1 and 2 outlined in the previous section, diffuse proper priors were placed on all remaining parameters in the model.

\section{Gemcitabine Pharmacogenomic Study}

Pancreatic cancer is a rapidly fatal disease with a 5-year survival rate of less than $5 \%[27,28]$. While gemcitabine is the standard chemotherapy used to treat pancreatic cancer, individuals' response to this drug varies widely. Genetic variation at each step within the gemcitabine metabolic pathway (transport, metabolism and drug target) could potentially influence the quantity of drug transported into the cell, metabolic inactivation of that drug, the rate of active drug formation and the quantity of active drug reaching its target(s).

To understand the pharmacogenomics of gemcitabine drug therapy, the Coriell Human Variation Panel lymphoblastoid cell lines were studied. Epstein Barr virus (EBV)-transformed B lymphoblastoid cells from three racial groups (white non-Hispanic Americans, African Americans and Han Chinese Americans) were purchased from the Coriell Institute (Camden, N.J., USA). Cytotoxicity assays were performed using the human lymphoblastoid cells treated at eight drug dosages of gemcitabine (e.g. $1,000,100,10,1,0.1,0.01,0.001$, and $0.0001 \mu \mathrm{M}$ ). Estimation of the quantitative phenotype IC50 (effective dose that kills 50\% of the 
Table 1. Genes included in bayesian hierarchical analysis

\begin{tabular}{|c|c|c|c|}
\hline Gene & Location & $\begin{array}{l}\text { Number of } \\
\text { SNPs in Genes }\end{array}$ & $\begin{array}{l}\text { Number of SNPs } \\
\text { included in analysis }\end{array}$ \\
\hline Cytidine deaminase (CDA) & 1p36.2-p35 & 17 & 16 \\
\hline Cytidine monophosphate kinase 1 (CMPK1) & $1 \mathrm{p} 32$ & 7 & 7 \\
\hline Deoxycytidine kinase (DCK) & $4 \mathrm{q} 13.3-\mathrm{q} 21.1$ & 22 & 22 \\
\hline 5'-nucleotidase, cytosolic IA (NT5C1A) & $1 \mathrm{p} 34.3-\mathrm{p} 33$ & 4 & 4 \\
\hline 5'-nucleotidase, cytosolic II (NT5C2) & $10 \mathrm{q} 24.32-\mathrm{q} 24.33$ & 12 & 12 \\
\hline 5'-nucleotidase, cytosolic III (NT5C3) & $7 \mathrm{p} 14.3$ & 10 & 8 \\
\hline 5'-nucleotidase, cytosolic III-like (NT5C3L) & $17 q 21.2$ & 4 & 3 \\
\hline $5^{\prime}, 3^{\prime}$-nucleotidase, mitochondrial (NT5M) & $17 \mathrm{p} 11.2$ & 23 & 23 \\
\hline Ribonucleotide reductase M1 (RRM1) & $11 \mathrm{p} 15.5$ & 21 & 20 \\
\hline Ribonucleotide reductase M2 polypeptide (RRM2) & $2 \mathrm{p} 25-\mathrm{p} 24$ & 9 & 9 \\
\hline $\begin{array}{l}\text { Solute carrier family } 28 \text { (sodium-coupled nucleoside transporter), } \\
\text { member } 2 \text { (SLC28A2) }\end{array}$ & $15 q 15$ & 12 & 11 \\
\hline Carrier family 29 (nucleoside transporters), member 1 (SLC29A1) & $6 \mathrm{p} 21.2-\mathrm{p} 21.1$ & 8 & 8 \\
\hline Solute carrier family 29 (nucleoside transporters), member 2 (SLC29A2) & $11 \mathrm{q} 13$ & 3 & 3 \\
\hline
\end{tabular}

${ }^{a}$ SNPs with Hardy-Weinberg equilibrium $\mathrm{p}>0.001$, call rate $>95 \%$ and minor allele frequency $>5 \%$.

cells) was completed using a four-parameter logistic model [29, 30].

Genotyping of SNPs using the Illumina HumanHap 550K BeadChips for the cell lines was completed at the Genotyping Shared Resources at the Mayo Clinic in Rochester, Minn., USA. Quality control was completed in which SNPs with Hardy-Weinberg equilibrium (HWE) p values $<0.001$ (minimum $\mathrm{p}$ value between exact test for $\mathrm{HWE}[31,32]$ and stratified test for HWE [33]), minor allele frequency $<5 \%$, or call rate $<95 \%$ were removed from further analyses. A total of 152 SNPs that passed quality control were identified in 13 genes contained within the gemcitabine pathway. Table 1 presents a breakdown of the number of SNPs in each of the 13 genes included in the statistical analysis. These SNPs were then analyzed using the four bayesian hierarchical models outlined in the two previous sections. Missing genotypes were imputed prior to all analyses using the program fastPHASE [34]. SNPs in perfect correlation with each other were removed prior to analysis in order to assure a positive definite covariance matrix.

\section{Description of Simulated Data}

Simulated genotype data was based on the gene COMT (Catechol-O-methyltransferase), which is important in the metabolism of catechol drugs used in the treatment of hypertension, asthma, and Parkinson's disease. Available genotypes for SNPs within COMT were downloaded for the HapMap CEU population (http:// www.hapmap.org/). Next, SNPs were tagged using ldSelect [35], with an $\mathrm{r}^{2}$ threshold of 0.64 and 0.80 and a minor allele frequency threshold of 0.05 . This was completed in order to remove redundant SNPs, which is routinely done in practice and to assess the impact of the amount of LD between markers on the method. A total of 42 SNPs were downloaded for COMT, with 12 and 17 SNP markers tagged at an $\mathrm{r}^{2}$ of 0.64 and 0.80 levels, respectively. Figure 1 displays the LD between SNP markers in COMT tagged at the $r^{2}=0.80$ level. The haplotype frequencies of this population were estimated using the haplo.em function within the haplo.stats R library (http://cran.r-project.org/web/packages/haplo.stats/index.html). These haplotype frequencies were then used as the 'true' haplotype frequency for the underlying population. One thousand haplotypes were simulated using the hapsim library in R (http://cran.r-project.org/web/packages/hapsim/index.html) based on the underlying 'true' haplotype frequencies from the HapMap CEU population. These haplotypes were then assigned in a sequential fashion to the 500 individuals.

Following the simulation of the genotypic data, the phenotypic data was simulated to mimic the phenotype in the gemcitabine pharmacogenomic study outlined in the accordingly named section. Phenotypic cytotoxicity values for each individual were simulated from a multivariate normal distribution $\operatorname{MVN}(\boldsymbol{\omega}, \boldsymbol{\psi})$ with mean based on the four parameter logistic function

$$
\boldsymbol{\omega}=\beta_{1}+\frac{\beta_{2}-\beta_{1}}{1+\exp \left\{\beta_{4}\left(\log (\mathbf{D})-\beta_{3}\right)\right\}},
$$

where the responses at infinite and zero concentration are represented by $\beta_{1}$ and $\beta_{2}$, respectively. The parameter $\beta_{3}$ represents the $\log ($ IC50), with IC50 representing the effective concentration that kills $50 \%$ of the cells. Lastly, $\beta_{4}$ represents the slope of the doseresponse curve. For the simulation of the phenotypic data, parameters in the model were set to $\beta_{1}=10, \beta_{2}=95$ and $\beta_{4}=1.5$.

For simulations in which there was a genetic effect on the phenotype $I C 50, \beta_{3}$ was set to $-3+($ effect size $) \times(A)$, with $A$ representing the number of minor alleles for a given SNP marker, while for the null simulations (no effect) $\beta_{3}$ was set to -3 . We simulated non-null data with two different effect sizes; 0.10 was to represent a small effect and 0.25 a large effect. Two different markers, in different regions of COMT, were used in the simulation of the 


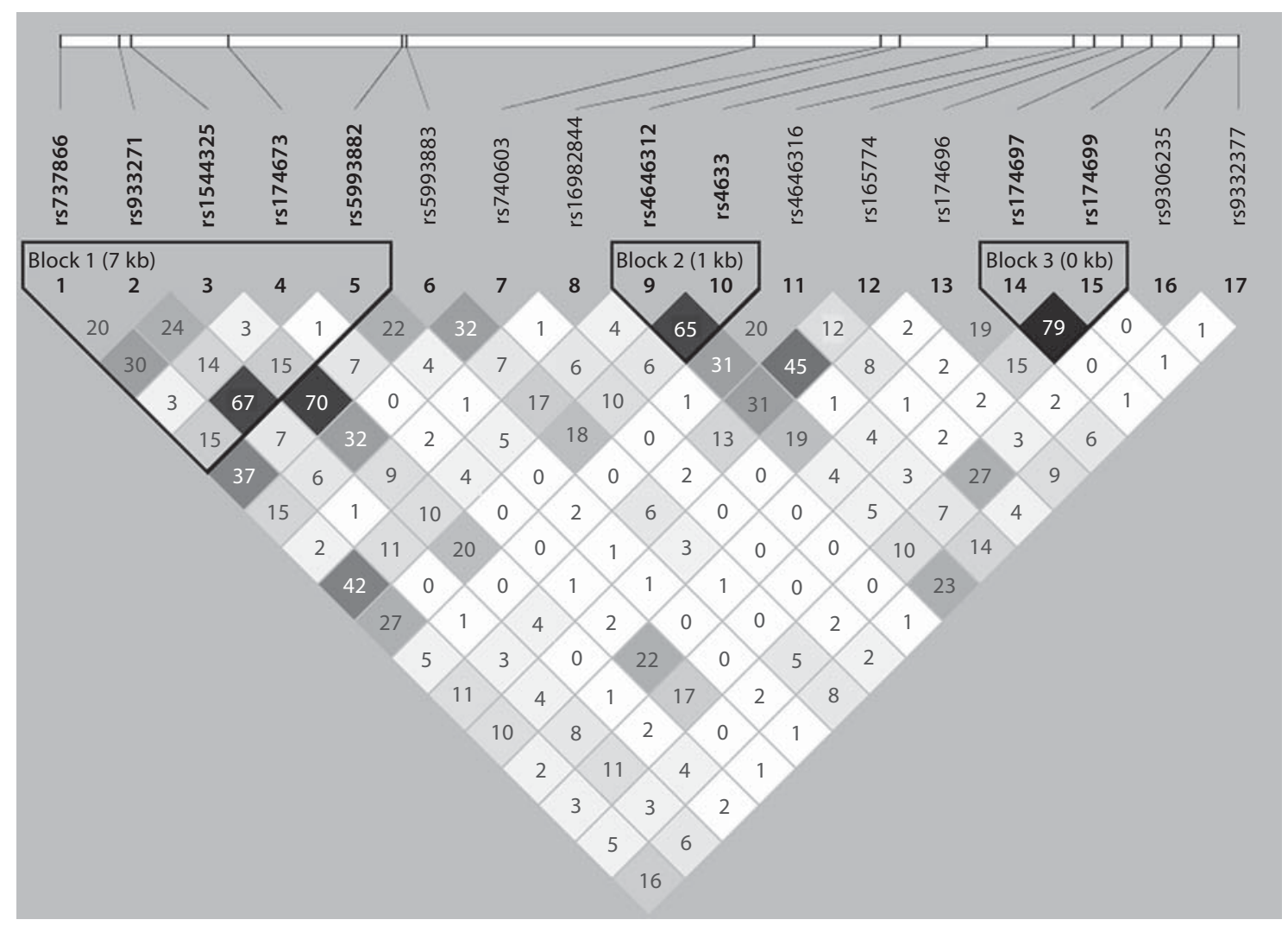

Fig. 1. LD plot for SNPs within COMT after tagging at $\mathrm{r}^{2}=0.80$, with values and colors representing LD between SNPs (measured with $\mathrm{r}^{2}$ ).

IC50 phenotype: rs1544325 and rs4646316 (fig. 1). The vector D contains the eight drug concentrations of $1,000,100,10,1,0.1$, $0.01,0.001$, and 0.0001 , selected to mimic the drug concentrations for the gemcitabine study and covariance matrix $\psi$ that has an autocorrelation structure with a lag of 1 , variance $\tau^{2}=100$ and autocorrelation parameter $\rho=0.65$. One hundred datasets for the eight non-null simulations (tagging at $\mathrm{r}^{2}$ of 0.80 or 0.64 and small or large SNP effect at either rs1544325 or rs464316) and two null simulations were generated, each containing 500 subjects (genotypes and IC50 phenotype).

\section{Results}

\section{Gemcitabine Pharmacogenomic Cytotoxicity Study}

The four hierarchical models, outlined in the two methods sections on hierarchical models, were applied to the gemcitabine pharmacogenomic study, described in the accordingly named section, to determine whether genetic variation within the gemcitabine pathway impacts the IC50 within 173 cell lines (60 white non-Hispanic Americans, 59 Han Chinese American, 54 African Amer- ican; 65 males and 108 females). For each of the 13 genes, the four models were fit using the WinBUGS software package (http://www.mrc-bsu.cam.ac.uk/bugs/welcome. shtml) [36] via the R package R2WinBUGS (http://cran.rproject.org/web/packages/R2WinBUGS/index.html) [37]. For each analysis, three independent chains were run, each with 55,000 iterations; the first 5,000 removed for burn-in and every 5th iteration thinned to save on storage space. In addition to SNP effects, race and gender effects were also included in each of the four models. Convergence of the Markov chain Monte Carlo was monitored and verified using the convergence measure $\sqrt{R}[38]$ and trace plots: the majority of $\sqrt{R}$ values were found to be less than 1.05 .

Comparing the results from the models where no LD information was included (model 1 vs. model 2), we observed little difference in estimated SNP effects (mean difference \pm standard deviation in differences: -0.0001 $\pm 0.0004)$ or length of $95 \%$ credible intervals $(-0.0002 \pm$ 0.004 ) between models with and without a gene-level effect. However, when LD information was included in the 


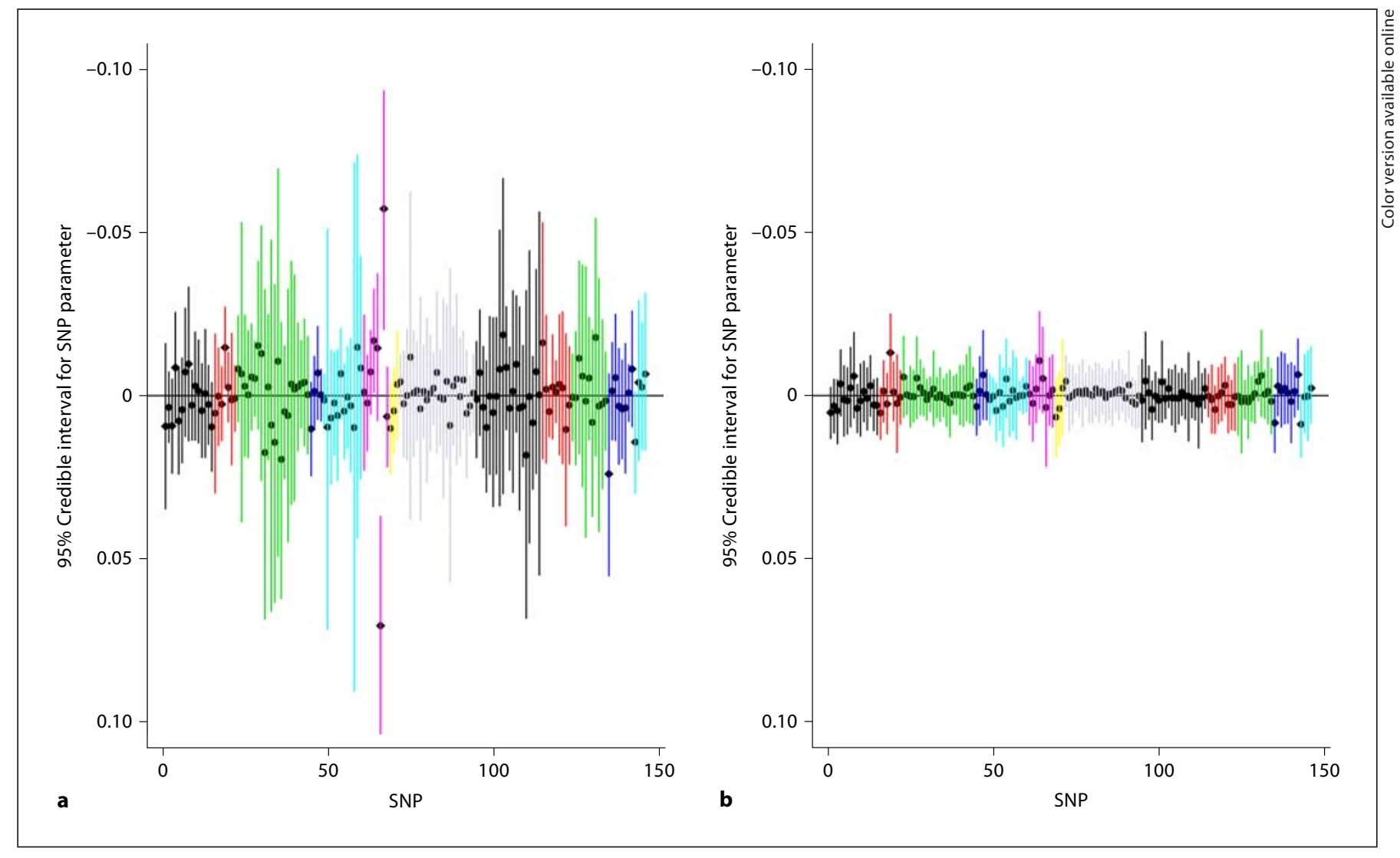

Fig. 2. Plots of $95 \%$ credible intervals for SNPs effect for the gemcitabine pharmacogenomic study with shrinkage of SNP effects towards 0; (a) LD information not included, (b) LD information included. The different colors of the intervals represent the different genes.

model (model 3 vs. 4), we did observe a moderate difference in the length of the $95 \%$ credible intervals $(-0.0025$ $\pm 0.004)$, with wider credible intervals observed for SNP effects based on a model that included a non-zero genelevel effect versus a model that shrank the SNP effects to zero. When LD information was included in the model, point estimates between the models were similar; however, length of $95 \%$ credible intervals was dramatically reduced (model 2 vs. model 4 with shrinkage to latent gene-level effect: $0.026 \pm 0.029$; model 1 vs. model 3 with shrinkage to zero: $0.028 \pm 0.029$ ) (fig. 2). Testing for gene-level association for each gene in the gemcitabine pathway with IC50 was completed based on the $95 \%$ credible interval for the parameter $\alpha$, with no gene-level associations detected.

In addition to fitting the four bayesian hierarchal models, we also completed a haplotype analysis using an expectation-maximation algorithm to estimate phase and a score test to assess association of the haplotypes with the phenotype IC50 $[39,40]$. Finally, the commonly used multi-SNP regression and stepwise regression models were fit to the data. An often overlooked issue in genetic association analysis is that regression analysis involving variables with high collinearity produces unstable parameter estimates and standard errors, and thus unstable $\mathrm{p}$ values for testing the individual contribution of each variable in the model [41]. Multiple regression models, however, are reliable for the assessment of the entire set of SNP variables in the model. Gene-level results from these multi-SNP analyses are presented in table 2. Comparing gene-level results between these frequentist multi-SNP models and our bayesian models, we observed that haplotype, linear regression and step-wise regression analyses all detected a gene-level effect for NT5C3, which was not detected with the bayesian analyses. 


\section{Simulation Studies}

The four bayesian hierarchical models were applied to the two simulated null scenarios and the four non-null simulation scenario datasets described in the methods section on simulated data. For every simulated dataset, each of the four models was fit using the WinBUGS software package [36] by way of the $\mathrm{R}$ package BRugs (http://www.mirrorservice.org/sites/lib.stat.cmu.edu/ R/CRAN/src/contrib/Descriptions/BRugs.html) [42]. For each analysis, three independent chains were run, each with 30,000 iterations, with the first 5,000 removed for burn-in. The point estimate for a parameter was taken to be the mean of the corresponding posterior distribution, with $95 \%$ credible intervals being the 2.5 th and the 97.5 th percentile of the posterior distribution. In addition to the bayesian models, single-SNP analysis using a Bonferroni or permutation approach to adjust for the multiple testing, haplotype analysis, multiple regression analysis and step-wise regression analysis were also completed.

The results of the simulation studies, pertaining to SNP effects and length of credible intervals, are presented in table 3 and figure 3 . Table 3 displays the number of simulations in which a SNP effect was detected based on $95 \%$ credible intervals with a simulated effect size of 0.25 (large) or 0.10 (small). For the null and non-null simulation scenarios, the four bayesian models performed equally well in terms of detecting the simulated 'causative' marker and false-positive SNP effects. For the simulation scenario with a large effect size (0.25), an $\mathrm{r}^{2}=0.80$ and a simulated effect at rs1544325, all four models were able to correctly detect the marker with a simulated effect. When the LD between markers was reduced with tagging at $r^{2}=0.64$ (effect size of 0.25 for rs1544325), all four models were able to correctly detect the simulated effect in $99 \%$ of the simulations. The markers with no effects simulated were detected in a modest number of simulations $(<12 \%)$, with most 'null' SNPs detected around $5 \%$ of the time. Similar results were also observed for simulated data tagging at $\mathrm{r}^{2}=0.80$ or $\mathrm{r}^{2}=0.64$ and with a simulated effect at rs4646316. Lastly, the model including both LD information and a latent gene-level effect (model 4) had fewer detected noncausative variants (less 'falsepositives') as compared to the other three models, as illustrated in table 4.

In comparison, commonly used methods for fitting single-SNP and multi-SNP models (i.e. multiple regression and step-wise regression) were unable to correctly determine the 'causative' effect from those markers with effects due to LD (table 5). The commonly used methods were able to detect the simulated 'causative' effect more
Table 2. Gene-level results for genes within the gemcitabine pathway

\begin{tabular}{lllll}
\hline Gene $^{\mathrm{a}}$ & \multicolumn{2}{l}{ Haplotype analysis } & $\begin{array}{l}\text { Linear } \\
\text { model } \\
\text { p value }\end{array}$ & $\begin{array}{l}\text { Stepwise } \\
\text { regression } \\
\text { p value }\end{array}$ \\
\cline { 2 - 3 } p value & d.f. & & 1.0 & 1.0 \\
\hline NT5M & 0.944 & 10 & 0.999 & 1.0 \\
RRM1 & 0.472 & 14 & 0.968 & 1.0 \\
NT5C2 & 0.52 & 10 & 0.675 & 1.0 \\
CDA & 0.592 & 13 & 0.999 & 1.0 \\
DCK & 0.314 & 12 & 0.898 & 1.0 \\
NT5C1A & 0.698 & 6 & 0.861 & 1.0 \\
SLC29A2 & 0.622 & 3 & 0.760 & 1.0 \\
RRM2 & 0.343 & 13 & 0.165 & 1.0 \\
SLC29A1 & 0.026 & 12 & 0.548 & 1.0 \\
CMPK & 0.516 & 12 & 0.699 & 1.0 \\
SLC28A2 & 0.125 & 9 & 0.001 & 0.001 \\
NT5C3 & 0.021 & 8 & 0.380 & 1.0 \\
NT5C3L & 0.23 & 3 &
\end{tabular}

a No gene-level effects were detected based on the $95 \%$ credible interval for the latent gene parameter, $\alpha$, from the four bayesian models.

b Based on final selected model; the only gene with SNPs selected and included in the final model was NT5C3, with 8 SNPs selected for the final model.

often than the bayesian hierarchal models but also detected more false-positive or 'null' effects. For the simulation scenarios with a small effect (0.10) at rs1544325, when tagged at $\mathrm{r}^{2}=0.80$, simulated effects were correctly detected using multiple regression and step-wise regression models in 84 and $83 \%$ of simulations, respectively. However, 'null' effects were detected in 71 and $94 \%$ of simulated datasets, with $29 \%$ of step-wise regression models detecting more than 3 SNPs. In addition to the joint modeling of all SNP markers, the single-SNP analyses, using permutation methods to determine empirical $\mathrm{p}$ values adjusted for multiple testing, were able to detect the causative variant in $69 \%\left(r^{2}=0.80\right)$ and $78 \%\left(r^{2}=0.64\right)$ of simulations. Nevertheless, just like the joint modeling methods, the single-SNP analysis approach detected noncausative effects in $43 \%\left(r^{2}=0.80\right)$ and $22 \%\left(r^{2}=0.64\right)$ of simulations.

Likewise, for the scenarios where a small effect for rs4646316 was simulated, when tagged at $r^{2}=0.80$, stepwise regression models correctly detected the simulated causative variant $71 \%$ of the time with $96 \%$ step-wise regression models detecting additional 'null' SNP markers (39\% of models detecting more than 3 SNPs). Analysis 


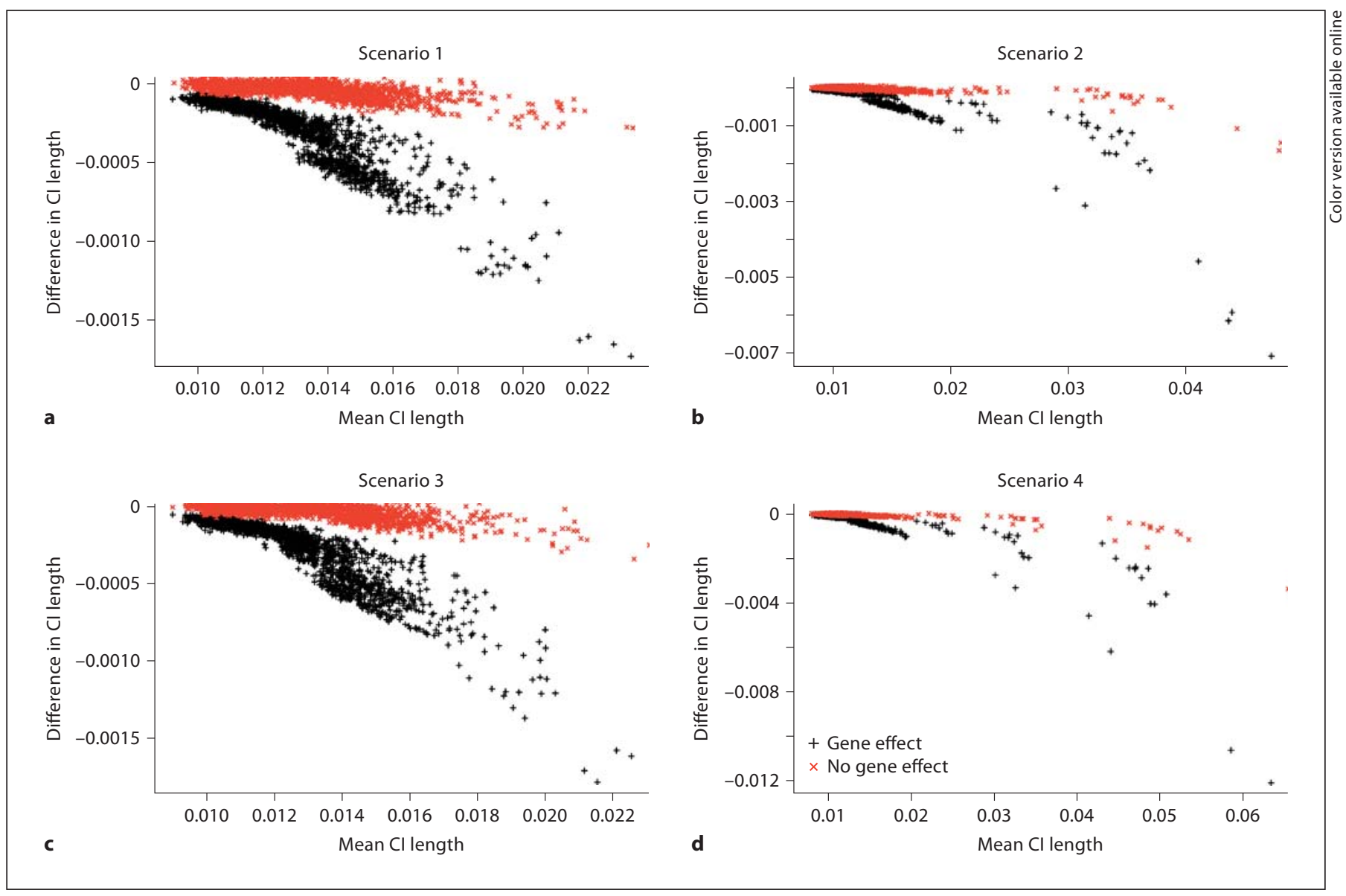

Fig. 3. Plots of the difference in credible interval lengths and mean interval lengths for inclusion and exclusion of LD information in the model for the four simulation scenarios based on a small effect (0.10) with ' + ' representing models with a gene effect included and ' $x$ ' representing models with no gene level effect. (a) Scenario with $r^{2}=0.80$ and effect at rs1544325. (b) Scenario with $r^{2}=0.64$ and effect at rs1544325. (c) Scenario with $r^{2}=$ 0.80 and effect at rs4646316. (d) Scenario with $\mathrm{r}^{2}=0.64$ and effect at $\mathrm{rs} 4646316$.

based on modeling each SNP individually detected the simulated effect less often than the joint modeling methods (57 and 56\% of simulations based on permutationbased $\mathrm{p}$ values), along with less percent of simulations detecting false-positives. Similar findings were observed for simulation scenarios with the effect size of 0.25 (data not shown).

No latent gene-level effects were detected for any of the null or non-null simulation scenarios using the bayesian hierarchical models. However, haplotype analysis was able to detect a global haplotype effect in $4-17 \%$ of the simulation scenarios with an effect size of 0.10 (table 5). In contrast, the step-wise regression and multiple regression models were able to detect a global gene-level effect in $94-97$ and $31-67 \%$ of simulations, respectively.

Localizing Putative Markers with

Bayesian Hierarchical Models
Lastly, we compared the parameter estimates and length of $95 \%$ credible intervals between the four bayesian models. No significant differences were observed in the SNP parameter estimates across the four models, with a maximum difference in parameter estimates less than $3.146 \times 10^{-4}$ for the simulations with a large simulated effect. Similar findings were also observed for the simulations with a small effect size. Figure 3 compares the length of credible intervals between models including or excluding LD information in the model for the four simulation scenarios with a small SNP effect (size $=0.10$ ). As the plots illustrate, the length of the credible intervals are slightly shorter when LD information is included in the model, especially for the case when a latent gene-level effect is also included in the model. 
Table 3. Number of simulations (out of 100) in which significant SNP effects were detected from each of the four models based on $95 \%$ credible intervals for simulations with effect size of $0.25(0.10)$.

\begin{tabular}{|c|c|c|c|c|c|c|c|c|c|c|c|c|c|}
\hline \multirow{3}{*}{$\begin{array}{l}\text { LD } \\
\left(r^{2}\right)\end{array}$} & \multirow[t]{3}{*}{ SNP } & \multicolumn{12}{|c|}{ Simulated SNP effect } \\
\hline & & \multicolumn{4}{|l|}{ Null } & \multicolumn{4}{|c|}{ rs 1544325} & \multicolumn{4}{|c|}{ rs4646316 } \\
\hline & & model 1 & model 2 & model 3 & model 4 & model 1 & model 2 & model 3 & model 4 & model 1 & model 2 & model 3 & model 4 \\
\hline \multirow[t]{17}{*}{0.80} & rs737866 & 6 & 6 & 5 & 4 & $6(5)$ & $7(5)$ & $6(5)$ & $4(5)$ & $5(5)$ & $5(5)$ & $5(5)$ & $5(5)$ \\
\hline & rs933271 & 3 & 3 & 3 & 3 & $5(3)$ & $5(3)$ & $5(3)$ & $5(3)$ & $4(4)$ & $4(4)$ & $4(3)$ & $2(3)$ \\
\hline & rs1544325 & 4 & 5 & 4 & 3 & $100(51)$ & $100(51)$ & $100(51)$ & $100(51)$ & $3(5)$ & $3(5)$ & $3(5)$ & $3(4)$ \\
\hline & rs174673 & 3 & 3 & 3 & 3 & $3(2)$ & $3(2)$ & $3(2)$ & $2(2)$ & $1(0)$ & $1(0)$ & $1(0)$ & $1(0)$ \\
\hline & rs5993882 & 2 & 2 & 2 & 2 & $1(1)$ & $1(1)$ & $1(1)$ & $1(1)$ & $3(2)$ & $3(2)$ & $3(2)$ & $3(2)$ \\
\hline & rs5993883 & 4 & 4 & 4 & 4 & $9(9)$ & $9(9)$ & $9(9)$ & $6(8)$ & $8(8)$ & $8(8)$ & $6(8)$ & $6(7)$ \\
\hline & rs740603 & 6 & 6 & 6 & 6 & $10(5)$ & $11(5)$ & $10(5)$ & $9(3)$ & $3(6)$ & $3(6)$ & $3(6)$ & $2(4)$ \\
\hline & rs16982844 & 1 & 1 & 1 & 1 & $7(4)$ & $7(4)$ & $7(4)$ & $7(4)$ & $4(4)$ & $4(4)$ & $4(4)$ & $4(3)$ \\
\hline & rs4646312 & 6 & 6 & 5 & 5 & $6(4)$ & $6(4)$ & $6(4)$ & $5(3)$ & $5(3)$ & $4(3)$ & $5(3)$ & $4(2)$ \\
\hline & rs4633 & 6 & 6 & 5 & 5 & $3(2)$ & $3(2)$ & $3(2)$ & $1(2)$ & $3(2)$ & $3(2)$ & $3(2)$ & $3(1)$ \\
\hline & rs4646316 & 5 & 5 & 5 & 4 & $3(4)$ & $3(4)$ & $3(4)$ & $3(4)$ & $100(57)$ & $100(57)$ & $100(57)$ & $100(57)$ \\
\hline & rs165774 & 4 & 4 & 4 & 4 & $8(7)$ & $8(7)$ & $8(6)$ & $9(6)$ & $8(6)$ & $8(6)$ & $8(6)$ & $6(6)$ \\
\hline & rs174696 & 4 & 5 & 4 & 4 & $9(9)$ & $9(9)$ & $9(9)$ & $8(8)$ & $8(4)$ & $9(5)$ & $8(5)$ & $8(4)$ \\
\hline & rs174697 & 6 & 6 & 6 & 3 & $6(3)$ & $6(3)$ & $5(3)$ & $2(2)$ & $2(6)$ & $2(6)$ & $2(6)$ & $1(2)$ \\
\hline & rs174699 & 3 & 3 & 3 & 2 & $3(2)$ & $3(2)$ & $3(2)$ & $1(2)$ & $2(4)$ & $2(4)$ & $2(4)$ & $2(3)$ \\
\hline & rs9306235 & 4 & 4 & 4 & 3 & $3(5)$ & $3(5)$ & $3(5)$ & $3(5)$ & $8(8)$ & $8(9)$ & $8(8)$ & $9(8)$ \\
\hline & rs9332377 & 6 & 5 & 4 & 4 & $5(4)$ & $5(4)$ & $5(4)$ & $5(3)$ & $5(4)$ & $5(4)$ & $5(4)$ & $6(4)$ \\
\hline \multirow[t]{12}{*}{0.64} & rs737866 & 5 & 5 & 4 & 2 & $3(4)$ & $3(4)$ & $3(4)$ & $1(1)$ & $4(4)$ & $4(4)$ & $4(4)$ & $4(2)$ \\
\hline & rs933271 & 6 & 5 & 5 & 5 & $4(5)$ & $4(5)$ & $4(5)$ & $4(5)$ & $4(4)$ & $4(4)$ & $4(4)$ & $4(4)$ \\
\hline & rs1544325 & 3 & 3 & 3 & 3 & $099(75)$ & $099(76)$ & 099 (75) & 099 (75) & $4(4)$ & $4(4)$ & $4(4)$ & $4(4)$ \\
\hline & rs174673 & 8 & 8 & 8 & 8 & $7(8)$ & $7(8)$ & $6(8)$ & $6(8)$ & $5(8)$ & $7(8)$ & $5(8)$ & $5(8)$ \\
\hline & rs16982844 & 7 & 7 & 7 & 7 & $8(7)$ & $6(7)$ & $6(7)$ & $6(7)$ & $7(7)$ & $6(7)$ & $6(7)$ & $6(7)$ \\
\hline & rs4646312 & 5 & 5 & 5 & 4 & $8(8)$ & $8(7)$ & $8(7)$ & $4(3)$ & $3(5)$ & $3(4)$ & $3(5)$ & $2(2)$ \\
\hline & rs4646316 & 8 & 8 & 7 & 7 & $6(8)$ & $6(8)$ & $5(7)$ & $5(7)$ & 099 (56) & $099(57)$ & 099 (57) & $099(57)$ \\
\hline & rs165774 & 3 & 3 & 3 & 3 & $6(4)$ & $5(4)$ & $6(4)$ & $6(4)$ & $3(3)$ & $3(3)$ & $3(3)$ & $3(3)$ \\
\hline & rs174696 & 5 & 5 & 5 & 4 & $6(5)$ & $6(5)$ & $6(5)$ & $6(5)$ & $4(5)$ & $4(6)$ & $4(6)$ & $3(4)$ \\
\hline & rs174697 & 5 & 5 & 5 & 5 & $6(5)$ & $5(5)$ & $6(5)$ & $4(4)$ & $5(5)$ & $5(5)$ & $5(5)$ & $4(5)$ \\
\hline & rs9306235 & 2 & 2 & 2 & 2 & $2(2)$ & $3(2)$ & $2(2)$ & $2(2)$ & $5(2)$ & $5(3)$ & $5(3)$ & $3(2)$ \\
\hline & rs9332377 & 9 & 9 & 9 & 8 & $10(9)$ & $10(9)$ & $9(9)$ & $9(9)$ & $11(11)$ & $1(10)$ & $11(11)$ & $11(9)$ \\
\hline
\end{tabular}

SNPs with simulated effects are in bold.

Table 4. Total number of non-null simulations (out of 100) for which an effect for a noncausative variant was detected

\begin{tabular}{|c|c|c|c|c|c|c|c|c|c|}
\hline \multirow[t]{3}{*}{$\mathrm{LD}\left(\mathrm{r}^{2}\right)$} & \multirow{3}{*}{$\begin{array}{l}\text { Effect } \\
\text { size }\end{array}$} & \multicolumn{8}{|c|}{ Simulated SNP effect } \\
\hline & & \multicolumn{4}{|c|}{ rs1544325 } & \multicolumn{4}{|c|}{ rs4646316 } \\
\hline & & model 1 & model 2 & model 3 & model 4 & model 1 & model 2 & model 3 & model 4 \\
\hline \multirow[t]{2}{*}{0.80} & 0.25 & 87 & 89 & 86 & 71 & 72 & 72 & 70 & 65 \\
\hline & 0.10 & 69 & 69 & 68 & 61 & 71 & 73 & 71 & 58 \\
\hline \multirow[t]{2}{*}{0.64} & 0.25 & 66 & 63 & 61 & 53 & 55 & 56 & 54 & 49 \\
\hline & 0.10 & 65 & 64 & 63 & 55 & 58 & 58 & 60 & 50 \\
\hline
\end{tabular}

The total numbers are given for each of the four models based on $95 \%$ credible intervals for simulations with effect size of 0.25 and 0.10 and $\mathrm{LD}$ of $\mathrm{r}^{2}=0.80$ and 0.64 . 
Table 5. Summary of simulations (out of 100) in which significant SNP effects were detected using haplotype, single and multiple regression and stepwise regression analysis methods with a simulated effect size of 0.10

\begin{tabular}{|c|c|c|c|c|c|}
\hline \multirow[t]{3}{*}{ Analysis method } & \multirow[t]{3}{*}{ Detected effects } & \multicolumn{4}{|c|}{ Marker with simulated effect } \\
\hline & & \multicolumn{2}{|c|}{ rs 1544325} & \multicolumn{2}{|c|}{ rs4646316 } \\
\hline & & $r^{2}=0.80$ & $\mathrm{r}^{2}=0.64$ & $\mathrm{r}^{2}=0.80$ & $r^{2}=0.64$ \\
\hline Haplotype & global haplotype effect & 17 & 8 & 10 & 4 \\
\hline \multirow[t]{3}{*}{ Single-SNP analysis ${ }^{1}$} & causative effect & 60 & 69 & 48 & 49 \\
\hline & noncausative effects & 26 & 17 & 24 & 16 \\
\hline & more than 2 SNPs with effects & 5 & 4 & 1 & 1 \\
\hline \multirow[t]{3}{*}{ Single-SNP analysis ${ }^{2}$} & causative effect & 69 & 78 & 57 & 56 \\
\hline & noncausative effects & 43 & 22 & 40 & 26 \\
\hline & more than 2 SNPs with effects & 7 & 5 & 5 & 4 \\
\hline \multirow[t]{4}{*}{ Multiple regression } & global gene effect & 39 & 67 & 31 & 46 \\
\hline & causative effect & 84 & 94 & 69 & 69 \\
\hline & noncausative effects & 71 & 64 & 74 & 63 \\
\hline & more than 2 SNPs with effects & 29 & 4 & 8 & 22 \\
\hline \multirow[t]{4}{*}{ Stepwise regression } & global gene effect & 97 & 98 & 96 & 94 \\
\hline & causative effect & 83 & 94 & 71 & 87 \\
\hline & noncausative effects & 94 & 87 & 96 & 88 \\
\hline & more than 3 SNPs with effects & 29 & 15 & 39 & 15 \\
\hline
\end{tabular}

\footnotetext{
${ }^{1}$ Significance based on Bonferroni adjusted significance level assuming all SNPs are independent.

${ }^{2}$ Significance based on permutation procedure to adjust for multiple testing taking into account the LD between SNPs.
}

\section{Discussion and Conclusions}

This paper presents an alternative method for completing multi-SNP analysis using a bayesian hierarchical model for assessing the effects of SNPs within the same gene (or region). We propose, when modeling the association of multiple SNPs in the same region or gene, that LD information be incorporated in the shrinkage of SNP effects to either a common latent gene-level effect or zero. The bayesian models can easily be modified for case-control studies in which a latent probit model [26] or a generalized linear model can be utilized. Lastly, the hierarchical models are applicable to both candidate gene or genome-wide association studies (GWASs). In the case of a GWAS, the SNPs would first be classified to one or several gene(s) or region(s) followed by the multi-SNP analysis using the bayesian hierarchical model.

Four different models were outlined and applied to a pharmacogenomic study of the drug gemcitabine and simulated data. We observed that the inclusion of LD information in the analysis resulted in no substantial dif- ferences in the point estimates of the SNP effects. However, including LD information did result in more precise parameter estimates (i.e. narrower credible intervals). This demonstrates that by utilizing the correlation structure existing in the genome, one is able to achieve more precise estimates of SNP effects. Additionally, the bayesian hierarchical model was able to isolate the simulated 'causative' variant more often than commonly used regression modeling methods. These observations corroborate previously reported research of an increase in precision for SNP parameter when LD information is included in the model [19] and the ability to isolate the putative variant associated with the phenotype using 'shrinkage' models [10]. These findings were observed for all simulation scenarios, with the largest gain in precision when LD between markers was high.

In addition to investigating the effect of including LD information in the model, we also assessed the ability to test for a 'gene' effect with the inclusion of a latent genelevel effect in the model. From the results of the simulation scenario in which a genetic effect was simulated 
through a SNP genotype (in terms of the number of minor alleles), the hierarchical model was unable to detect a global gene effect. Future research is needed to determine how to increase the power to detect gene-level effects via the multi-SNP hierarchical models. However, if the 'true' genetic effect is due to a haplotype as opposed to a SNP, we may be unable to detect a haplotype genetic effect using the proposed hierarchical models with a latent genelevel effect. The lack of inclusion of phase information is also a limitation of other multiple SNP analysis approaches (e.g. shrinkage, variable selection, and data reduction methods).

In conclusion, incorporation of $\mathrm{LD}$ information in the analysis of multiple SNPs results in smaller credible intervals and therefore more precise estimates of SNP effects, as illustrated in both the gemcitabine pharmacogenomic study and the simulation study. We have chosen to model the LD based on the observed $\mathrm{r}^{2}$ between markers Further research is needed to assess different models for including LD (e.g. $D^{\prime}, r^{2}$ estimates based on HapMap, spatial dependency, and LD graphs [19]). In simulation sce- narios in which a genetic effect was simulated, smaller intervals were observed when SNP effects were shrunk to a latent gene-level effect as opposed to zero, when LD information was included in the model. Otherwise, little difference in results was observed between the model that shrank SNP effects to zero and the model that shrank SNP effects to a latent gene level effect. Finally, based on our simulation study, the bayesian hierarchical model was able to isolate the marker with the simulated effect more often than commonly used regression modeling methods.

\section{Acknowledgements}

We would like to thank Dr. Liewei Wang for use of data from a pharmacogenomic study of gemcitabine. The research was supported by the NIH grants U01 GM61388, and R21 CA140879, a Mayo Clinic Cancer Center Eagle's Award, and a Minnesota Partnership for Biotechnology and Medical Genomics grant H9046000431.

\section{References}

1 Cordell HJ, Clayton DG: Genetic association studies. Lancet 2005;366:1121-1131.

-2 Hirschhorn JN, Daly MJ: Genome-wide association studies for common diseases and complex traits. Nat Rev Genet 2005;6:95108 .

-3 Hindorff LA, Sethupathy P, Junkins HA, Ramos EM, Mehta JP, Collins FS, Manolio TA: Potential etiologic and functional implications of genome-wide association loci for human diseases and traits. Proc Natl Acad Sci USA 2009;106:9362-9367.

-4 Pettipher R, Cardon LR: The application of genetics to the discovery of better medicines. Pharmacogenomics 2002;3:257-263.

5 Piquette-Miller M, Grant DM: The art and science of personalized medicine. Clin Pharmacol Ther 2007;81:311-315

6 Nebert DW, Vesell ES: Advances in pharmacogenomics and individualized drug therapy: exciting challenges that lie ahead. Eur J Pharmacol 2004;500:267-280.

7 Weinshilboum R: Inheritance and drug response. N Engl J Med 2003;348:529-537.

$\checkmark 8$ Weinshilboum R, Wang L: Pharmacogenomics: bench to bedside. Nat Rev Drug Discov 2004;3:739-748.

$\checkmark 9$ Weinshilboum RM, Wang L: Pharmacogenetics and pharmacogenomics: development, science, and translation. Annu Rev Genomics Hum Genet 2006;7:223-245.
10 Malo N, Libiger O, Schork NJ: Accommodating linkage disequilibrium in genetic-association analyses via ridge regression. Am J Hum Genet 2008;82:375-385.

11 Tibshirani R: Regression shrinkage and selection via the lasso. J R Stat Soc Series B Stat Methodol 1996;58:267-288.

12 George EI, McCulloch RE: Variable selections via gibbs sampling. J Am Stat Assoc 1993;88:881-889.

-13 Fridley BL: Bayesian variable and model selection methods for genetic association studies. Genet Epidemiol 2008;32:52-60.

14 Lunn DJ, Whittaker JC, Best N: A bayesian toolkit for genetic association studies. Genet Epidemiol 2006;30:231-247.

15 Witte JS: Genetic analysis with hierarchical models. Genet Epidemiol 1997;14:11371142.

16 Bryk AS, Raudenbush SW: Hierarchical Linear Models: Applications and Data Analysis Methods. Newbury Park, CA, SAGE Publications, Inc., 1992.

17 Snijders TAB, Bosker RJ: Multilevel Analysis. London, SAGE Publications Ltd, 1999.

18 Conti DV, Witte JS: Hierarchical modeling of linkage disequilibrium: genetic structure and spatial relations. Am J Hum Genet 2003; 72:351-363.

19 Li H, Wei Z, Maris J: A hidden Markov random field model for genome-wide association studies. Biostatistics 2010;11:139-150.
20 Hsiang T: A bayesian view on ridge regression. Statistician 1975;24:267-268

21 Browne WJ, Draper D: Implementation and performance issues in the bayesian and likelihood fitting of multilevel models. Comput Stat 2000;15:391-420.

22 Raftery AE: Bayesian model selection in social research. Sociol Methodol 1995;25:111163.

23 Conti DV, Cortessis V, Molitor J, Thomas DC: Bayesian modeling of complex metabolic pathways. Hum Hered 2003;56:83-93.

-24 Viallefont V, Raftery AE, Richardson S: Variable selection and bayesian model averaging in case-control studies. Stat Med 2001; 20:3215-3230.

25 Hoeting JA, Madigan D, Raftery AE, Volinsky CT: Bayesian model averaging: a tutorial. Stat Sci 1999;14:382-417.

26 Albert JH, Chib S: Bayesian analysis of binary and polychotomous response data. J Am Stat Assoc 1993;88:669-679.

-27 Jemal A, Murray T, Ward E, Samuels A, Tiwari RC, Ghafoor A, Feuer EJ, Thun MJ: Cancer statistics, 2005. CA Cancer J Clin 2005;55:10-30.

28 Li D, Xie K, Wolff R, Abbruzzese JL: Pancreatic cancer. Lancet 2004;363:1049-1057.

29 Davidian M, Giltinan DM: Nonlinear Models for Repeated Measurement Data. New York, Chapman \& Hall, 1995. 
30 Gallant AR: Nonlinear statistical models. New York, Wiley, 1987.

31 Guo SW, Thompson EA: Performing the exact test of Hardy-Weinberg proportion for multiple alleles. Biometrics 1992;48:361372.

- 32 Wigginton JE, Cutler DJ, Abecasis GR: A note on exact tests of Hardy-Weinberg equilibrium. Am J Hum Genet 2005;76:887-893.

- 33 Schaid DJ, Batzler AJ, Jenkins GD, Hildebrandt MA: Exact tests of Hardy-Weinberg equilibrium and homogeneity of disequilibrium across strata. Am J Hum Genet 2006; 79:1071-1080.
34 Scheet P, Stephens M: A fast and flexible statistical model for large-scale population genotype data: applications to inferring missing genotypes and haplotypic phase. Am J Hum Genet 2006;78:629-644.

-35 Carlson CS, Eberle MA, Rieder MJ, Yi Q, Kruglyak L, Nickerson DA: Selecting a maximally informative set of single-nucleotide polymorphisms for association analyses using linkage disequilibrium. Am J Hum Genet 2004;74:106-120.

36 Spiegelhalter D, Thomas A, Best N, Lunn D: WinBUGS version 2.0 User Manual. Cambridge, MRC Biostatistics Unit, 2004.

-37 Sturtz S, Ligges U, Gelman A: R2WinBUGS: A package for running WinBUGS from R. J Stat Softw 2005;12:1-16.

38 Gelman A, Carlin JB, Stern HS, Rubin DB: Bayesian Data Analysis. London, Chapman \& Hall, 1995.
39 Schaid DJ, Rowland CM, Tines DE, Jacobson RM, Poland GA: Score tests for association between traits and haplotypes when linkage phase is ambiguous. Am J Hum Genet 2002; 70:425-434.

40 Excoffier L, Slatkin M: Maximum-likelihood estimation of molecular haplotype frequencies in a diploid population. Mol Biol Evol 1995;12:921-927.

41 Neter J, Kutner MH, Nachtsheim CJ, Wasserman W: Applied Linear Statistical Models, ed 4. McGraw-Hill Companies, Inc., 1996.

42 Thomas A: BRugs User Manual, version 1.0. Dept of Mathematics \& Statistics, University of Helsinki, 2004. 